\title{
Ethylene Oxide Sterilization
}

National Cancer Institute

\section{Source}

National Cancer Institute. Ethylene Oxide Sterilization. NCI Thesaurus. Code C101713.

A sterilization process that uses ethylene oxide gas to kill microorganisms. 